\title{
Electric mobility, renewable energies and climate protection - challenges for our infrastructures
}

Volker Blandow, TÜV SÜD AG

This manuscript was not available on completion of this publication.

Thank you for your understanding. 